\section{TATRA \\ MOUNTaiNS \\ Mathematical Publications}

DOI: $10.2478 / \mathrm{tmmp}-2021-0019$

Tatra Mt. Math. Publ. 79 (2021), 47-58

\title{
EXPLICIT NON ALGEBRAIC LIMIT CYCLE FOR A DISCONTINUOUS PIECEWISE DIFFERENTIAL SYSTEMS SEPARATED BY ONE STRAIGHT LINE AND FORMED BY LINEAR CENTER AND LINEAR SYSTEM WITHOUT EQUILIBRIA
}

\author{
AzIZA BerbaCHE \\ Department of Mathematics, Faculty of Mathematics and Informatics, \\ University of Bordj Bou Arréridj, El Anasser, ALGERIA
}

\begin{abstract}
In this paper, we deal with the discontinuous piecewise differential linear systems formed by two differential systems separated by a straight line when one of these two differential systems is a linear without equilibria and the other is a linear center. We are going to show that the maximum number of crossing limit cycles is one, and if exists, it is non algebraic and analytically given.
\end{abstract}

\section{Introduction and statement of main result}

An important problem of the qualitative theory of differential equations is to determine the limit cycles of differential equations in the plane. We usually only ask for the number of such limit cycles, but their location as orbits of the system is also an interesting problem, and an even more difficult problem is to give an explicit expression of them. In the last few years, there has been an increasing interest in the study of piecewise differential systems, see [2, 5, 13, 14, 16, 19] for instance. This interest has been mainly motivated by their wide range of application in modelling real phenomena (e.g., control theory, biology, chemistry, engineering, physics).

(C) 2021 Mathematical Institute, Slovak Academy of Sciences.

2010 Mathematics Subject Classification: 34A30, 34C05, 34C25, 34C07.

Keyw ords: limit cycle, first integral, discontinuous piecewise linear differential system.

(ㄷ) (1) @ $\Theta$ Licensed under the Creative Commons BY-NC-ND 4.0 International Public License. 
The simplest possible piecewise linear differential systems are the ones having only two pieces separated by a straight line in the plane $\mathbb{R}^{2}$. In 1990 , Lum and Chua conjectured in [22, 23] that such continuous piecewise linear differential systems have at most one limit cycle. In 1998, this conjecture was proved by Freire et al. 9]. In 2013, a new and shorter proof was done by Llibre, Ordonez and E. Ponce [19].

Limit cycles of discontinuous piecewise linear differential systems separated by a straight line have been studied by many authors, see for instance [1, 10, 12, 14, 15, 19] and the references therein. There are examples of such systems exhibiting three limit cycles, see [4, 5, 13, 16, 17, and at this moment we do not know if discontinuous piecewise linear differential systems separated by a straight line can have more than three limit cycles. The case when the two linear differential systems which form the piecewise linear differential system have no equilibrium points was studied in Llibre and Teixeira [20] in 2016, where it is proved that the continuous piecewise systems have no limit cycles, but the discontinuous piecewise system can have at most one limit cycle. Euzébio and Llibre [7] proved that if one of the two linear differential systems has its equilibrium points on the line of discontinuity, then the discontinuous piecewise system has at most four limit cycles. In [18] and later on in 21, it is proved that if one of the two linear differential systems of the discontinuous piecewise linear differential system is a center; the maximum number of crossing limit cycles is two. However in all these papers, explicit non-algebraic limit cycles do not appear. As that the authors also forgot to analyse the case when one of the two linear differential systems which forms the piecewise linear differential system has no equilibrium points.

In this paper, we are interested in the discontinuous piecewise linear differential systems separated by one straight line with two linear systems, such that one of them is a linear center and the second one has no equilibria, neither real nor virtual. Moreover, the main purpose of this paper is to investigate the existence, number and the explicit expression of crossing period orbits for these systems.

We assume without loss of generality the two pieces in the plane, where the discontinuous piecewise linear differential systems are the left and the right halfplanes

$$
\Sigma_{-}=\left\{(x, y) \in \mathbb{R}^{2}: x<0\right\}, \quad \Sigma_{+}=\left\{(x, y) \in \mathbb{R}^{2}: x>0\right\} .
$$

Consequently, $\Sigma=\left\{(x, y) \in \mathbb{R}^{2}: x=0\right\}$ is the straight line of separation between two linear differential systems.

In order to state precisely our results, we introduce some notations and definitions. Consider the piecewise differential system

$$
X_{ \pm}=(\dot{x}, \dot{y})=\left(f_{ \pm}(x, y), g_{ \pm}(x, y)\right)
$$

defined in $\Sigma_{ \pm}$, respectively. We will use the techniques and approaches presented by Filippov in [8] and by di Bernardo et al. [6] to establish these notations. 


\section{NON ALGEBRAIC LIMIT CYCLE FOR A CLASS OF PIECEWISE LINEAR SYSTEMS}

If an equilibrium point of $X_{ \pm}$is in $\Sigma_{ \pm}$, it is said that it is a real equilibrium point for the piecewise differential system. Otherwise, it is called a virtual equilibrium point. In order to extend the definition of a trajectory to $\Sigma$, we split $\Sigma$ into three parts depending on whether or not the vector field points towards it:

1. Crossing region:

$$
\Sigma_{c}=\left\{p \in \Sigma: f_{+}(p) f_{-}(p)>0\right\} .
$$

2. Attractive sliding region:

$$
\Sigma_{a s}=\left\{p \in \Sigma: f_{+}(p)<0 \text { and } f_{-}(p)>0\right\} .
$$

3. Repulsive sliding region:

$$
\Sigma_{r s}=\left\{p \in \Sigma: f_{+}(p)>0 \text { and } f_{-}(p)<0\right\} .
$$

These three regions are relatively open in $\Sigma$ and can have several connected components. Therefore, their definitions exclude the so-called tangency points, that is, points where one of the two vector fields is tangent to $\Sigma$, which can be characterized by

$$
\left\{p \in \Sigma: f_{+}(p)=0 \quad \text { or } \quad f_{-}(p)=0\right\} .
$$

These points are on the boundary of the regions $\Sigma_{c}, \Sigma_{a s}$ and $\Sigma_{r s}$.

Periodic orbits that have neither sliding part nor tangent points are called crossing periodic orbits, otherwise they are called sliding periodic orbits.

It is known that if the vector field has no equilibrium points, it can be written as

$$
\dot{x}=a x+b y+c, \quad \dot{y}=\mu a x+\mu b y+d,
$$

where $a, b, c, \mu$ and $d$ are real constants such that $d \neq \mu c$ and $\mu \neq 0$.

In the following, we need a lemma on the explicit expression of the first integral of an arbitrary linear differential system without equilibrium points. The following lemma is presented in [3].

LEMMA 1.1. For system (1) the following statements hold.

i) If $a+b \mu=0$, then system (11) is Hamiltonian and all its solutions are algebraic and given by parabolas. Moreover, this system has the first integral

$$
H(x, y)=\frac{1}{2}\left(b \mu^{2} x^{2}-2 b \mu x y-2 d x+b y^{2}+2 c y\right) .
$$

ii) If $a+b \mu \neq 0$ and $d-c \mu \neq 0$, the only algebraic invariant curve of (1) is an invariant line. Moreover, this system has the first integral

$$
H(x, y)=((a+b \mu)(a x+b y)+a c+b d) e^{\frac{a+b \mu}{d-c \mu}(\mu x-y)} .
$$


It is known that (see 20]) a normal form for a linear differential system having a center is given in the next result.

Lemмa 1.2. A linear differential system having a center can be written as

$$
\dot{x}=-B x-\frac{4 B^{2}+w^{2}}{4 A} y+\delta, \quad \dot{y}=A x+B y+D,
$$

with $A \neq 0$ and $w>0$. This linear differential system has the firstintegral

$$
H_{1}(x, y)=4(A x+B y)^{2}+8 A(D x-\delta y)+w^{2} y^{2} .
$$

Our main results are the following.

TheOREM 1.3. A discontinuous piecewise linear differential system separated by one straight line with two linear systems, such that one of them is a linear center and the second have no equilibria, neither real nor virtual, can have at most one limit cycle. Moreover, this limit cycle if there exists is hyperbolic and non algebraic.

Theorem 1.3 is proved in Section 2.

The next proposition shows that there are discontinuous piecewise linear differential systems separated by the straight line $\Sigma$ with two linear systems such that one of them is a linear center and the second one has no equilibria, neither real nor virtual, with one crossing non algebraic limit cycle.

Proposition 1.4. For

$$
\begin{aligned}
& a=\mu-1, \quad c=-\frac{1}{2}, \quad d=-\frac{1}{2} \mu-\frac{1}{2}, \quad b=-1, \\
& A=\frac{5}{16}, \quad \delta=-1, \quad B=1, \quad D=1 \quad \text { and } \quad w=1
\end{aligned}
$$

the discontinuous piecewise linear differential system defined by

$$
\begin{array}{lll}
\dot{x}=-x-4 y-1, & \dot{y}=\frac{5}{16} x+y+1 & \text { in } \Sigma_{+}, \\
\dot{x}=(\mu-1) x-y-\frac{1}{2}, & \dot{y}=\mu(\mu-1) x-\mu y-\frac{1}{2}(\mu+1), & \text { in } \Sigma_{-},
\end{array}
$$

when $\mu \neq 0$ has one explicit hyperbolic non-algebraic crossing limit cycle given by

$$
\begin{gathered}
\Gamma=\left\{(x, y) \in \Sigma_{+}: 4\left(\frac{5}{16} x+y\right)^{2}+\frac{5}{2}(x+y)+y^{2}=1.7607\right\} \cup \\
\left\{(x, y) \in \Sigma_{-}:((1-\mu) x+y+1) e^{2 \mu x-2 y}=0.63399\right\} .
\end{gathered}
$$

This proposition will be proved in Section 3 . 


\section{Proof of Theorem 1.3}

Assume that we have a discontinuous piecewise linear differential system separated by one straight line and formed by two linear systems such that one of them is a linear center and the second one has no equilibria, neither real nor virtual. By Lemma 1.2 and using the normal form (10), we can write such a discontinuous piecewise linear differential system as

$$
\begin{array}{lll}
\dot{x}=-B x-\frac{4 B^{2}+w^{2}}{4 A} y+\delta, & \dot{y}=A x+B y+D, & \text { in } \Sigma_{+}, \\
\dot{x}=a x+b y+c, & \dot{y}=\mu a x+\mu b y+d, & \text { in } \Sigma_{-} .
\end{array}
$$

Notice that system (4) in the half-plane $\Sigma_{+}$has the first integral

$$
H_{1}(x, y)=4(A x+B y)^{2}+8 A(D x-\delta y)+w^{2} y^{2} .
$$

By lemma 1.1, the first integral of discontinuous piecewise linear differential system (44) in the half-plane $\Sigma_{-}$, is

$$
H_{2}(x, y)= \begin{cases}((a+b \mu)(a x+b y)+a c+b d) e^{\frac{a+b \mu}{d-c \mu}(\mu x-y)} & \text { if } a+b \mu \neq 0 \\ \frac{1}{2}\left(b \mu^{2} x^{2}-2 b \mu x y-2 d x+b y^{2}+2 c y\right) & \text { if } a+b \mu=0 .\end{cases}
$$

Suppose that this of discontinuous piecewise linear differential system (41) has some limit cycles intersecting $\Sigma$ in two points, namely, $\left(0, y_{0}\right)$ and $\left(0, y_{1}\right)$ with $y_{1} \neq y_{0}$. Then, the first integrals $H_{1}$ and $H_{2}$ must satisfy the following two equations:

$$
\begin{aligned}
& H_{1}\left(0, y_{0}\right)-H_{1}\left(0, y_{1}\right)=0, \\
& H_{2}\left(0, y_{0}\right)-H_{2}\left(0, y_{1}\right)=0 .
\end{aligned}
$$

Note that the first equation of (15) can be written as

$$
\left(4 B^{2}+w^{2}\right) y_{0}^{2}-8 A \delta y_{0}-\left(4 B^{2}+w^{2}\right) y_{1}^{2}+8 A \delta y_{1}=0 .
$$

Taking into account that we are only interested in the solutions $y_{1}$ satisfying $y_{1} \neq y_{0}$, the unique solution of the previous equation is

$$
y_{1}=\frac{8 A \delta}{4 B^{2}+w^{2}}-y_{0}
$$

Now, it is easy to see that the existence of crossing periodic solutions of discontinuous piecewise linear differential system (4) is equivalent to the existence of the isolated values of $y_{0}$ which satisfied

$$
H_{2}\left(0, y_{0}\right)-H_{2}\left(0, \frac{8 A \delta}{4 B^{2}+w^{2}}-y_{0}\right)=0 \text {. }
$$




\section{AZIZA BERBACHE}

Here, we have to divide the proof of Theorem 1.3 into two cases.

Case 1. if $a+\mu b=0$ and $d-c \mu \neq 0$ : In this case (17) becomes

$$
\frac{4\left(4 B^{2} c+c w^{2}+4 A b \delta\right)}{\left(4 B^{2}+w^{2}\right)^{2}}\left(\left(4 B^{2}+w^{2}\right) y_{0}-4 A \delta\right)=0 .
$$

It is easy to see when $4 B^{2} c+c w^{2}+4 A b \delta=0$, this equation has infinity solution $y_{0}$. So the periodic orbits of the discontinuous piecewise linear differential system (44) are in a continuum of periodic orbits, and consequently the discontinuous piecewise linear differential system (4) has no limit cycles. If

$$
4 B^{2} c+c w^{2}+4 A b \delta \neq 0,
$$

the unique solution of (8) is $y_{0}=\frac{4 A \delta}{4 B^{2}+w^{2}}$. From this value of $y_{0}$ we get the value of $y_{1}=\frac{4 A \delta}{4 B^{2}+w^{2}}$, so $y_{0}=y_{1}$, which cannot contribute a limit cycle and consequently, the discontinuous piecewise linear differential system (4) has no limit cycles. This completes the proof of Theorem 1.3 in Case 1.

Case 2. if $a+\mu b \neq 0$ and $d-c \mu \neq 0$ : In this Case (17) becomes

$$
\begin{aligned}
\left(b(a+b \mu)\left(\frac{8 A \delta}{4 B^{2}+w^{2}}-y_{0}\right)+\right. & a c+b d) e^{-\frac{a+b \mu}{d-c \mu}\left(\frac{8 A \delta}{4 B^{2}+w^{2}}-y_{0}\right)} \\
& =\left(b(a+b \mu) y_{0}+a c+b d\right) e^{-\frac{a+b \mu}{d-c \mu} y_{0}}
\end{aligned}
$$

This last equation can be written as

$$
\begin{aligned}
0= & \left(b(a+b \mu) y_{0}+a c+b d\right) \\
& -\left(b(a+b \mu)\left(\frac{8 A \delta}{4 B^{2}+w^{2}}-y_{0}\right)+a c+b d\right) e^{-\frac{a+b \mu}{d-c \mu}\left(\frac{8 A \delta}{4 B^{2}+w^{2}}-2 y_{0}\right)} .
\end{aligned}
$$

For convenience, we denote by

$$
\begin{aligned}
f(y)= & (b(a+b \mu) y+a c+b d) \\
& -\left(b(a+b \mu)\left(\frac{8 A \delta}{4 B^{2}+w^{2}}-y\right)+a c+b d\right) e^{-\frac{a+b \mu}{d-c \mu}\left(\frac{8 A \delta}{4 B^{2}+w^{2}}-2 y\right)} .
\end{aligned}
$$

Now, solving (9) is equivalent to find the solutions $y_{0 i}$ of the equation $f(y)=0$. In order to investigate the number of solutions of $f(y)=0$, and since $f$ is a function differentiable in $\mathbb{R}$, we shall use the first two derivatives of the function $f$. Simple calculations yield:

$$
\begin{aligned}
f^{\prime}(y)= & \frac{-(a+b \mu)}{\left(4 B^{2}+w^{2}\right)(d-c \mu)} b\left(4 B^{2}+w^{2}\right)(c \mu-d) \\
& -\left(\left(4 B^{2}+w^{2}\right)((2 a c+b d+b c \mu)-2 b(a+b \mu) y)+16 A b \delta(a+b \mu)\right) \\
& \times \frac{(a+b \mu)}{\left(4 B^{2}+w^{2}\right)(d-c \mu)} e^{-\frac{a+b \mu}{d-c \mu}\left(\frac{8 A \delta}{4 B^{2}+w^{2}}-2 y\right)},
\end{aligned}
$$


NON ALGEBRAIC LIMIT CYCLE FOR A CLASS OF PIECEWISE LINEAR SYSTEMS

$$
\begin{aligned}
f^{\prime \prime}(y)=-4 \frac{(a+b \mu)^{3}}{\left(4 B^{2}+w^{2}\right)(d-c \mu)^{2}}\left(\left(4 B^{2}+w^{2}\right)(c-\right. & b y)+8 A b \delta) \\
& \times e^{2 \frac{a+b \mu}{d-c \mu}\left(y-\frac{4 A \delta}{4 B^{2}+w^{2}}\right)} .
\end{aligned}
$$

It is easy to see that $f^{\prime}$ and $f^{\prime \prime}$ are continuous functions in $\mathbb{R}$. It is obvious that $f^{\prime \prime}(y)=0$ has at most one root, it follows that the function $f^{\prime}$ has at most two zeros. Thus $f(y)=0$ has at most three roots $y_{0 i}, i=1,2,3$. It is easy to see that $y=\frac{4 A \delta}{4 B^{2}+w^{2}}$ is a solution of the equation $f(y)=0$.

On the other hand, we have

$$
\begin{aligned}
f\left(\frac{8 A \delta}{4 B^{2}+w^{2}}-y_{0}\right)= & \left(b(a+b \mu)\left(\frac{8 A \delta}{4 B^{2}+w^{2}}-y_{0}\right)+a c+b d\right) \\
& -\left(b(a+b \mu) y_{0}+a c+b d\right) e^{\frac{a+b \mu}{d-c \mu}\left(\frac{8 A \delta}{4 B^{2}+w^{2}}-2 y_{0}\right)} .
\end{aligned}
$$

The equation $f\left(\frac{8 A \delta}{4 B^{2}+w^{2}}-y_{0}\right)=0$ can be written as

$$
\begin{aligned}
\left(b(a+b \mu)\left(\frac{8 A \delta}{4 B^{2}+w^{2}}-y_{0}\right)\right. & +a c+b d) \\
& -\left(b(a+b \mu) y_{0}+a c+b d\right) e^{\frac{a+b \mu}{d-c \mu}\left(\frac{8 A \delta}{4 B^{2}+w^{2}}-2 y_{0}\right)}=0,
\end{aligned}
$$

this is equivalent to $f\left(y_{0}\right)=0$. Therefore, if $y_{0}$ is a solution of $f(y)=0$, then $\frac{8 A \delta}{4 B^{2}+w^{2}}-y_{0}$ is also a solution of this last equation. Thus, three solutions of the equation $f(y)=0$ (if there exist) are:

- $y_{01}=\frac{4 A \delta}{4 B^{2}+w^{2}}$, by (6) and from this value of $y_{01}$ we get the value of $y_{11}=\frac{4 A \delta}{4 B^{2}+w^{2}}$, so $y_{01}=y_{11}$, which cannot contribute a limit cycle.

- $y_{02}=y_{0}$, by (6) and from this value of $y_{02}$ we get the value of $y_{12}=\frac{8 A \delta}{4 B^{2}+w^{2}}-y_{0}$.

- $y_{03}=\frac{8 A \delta}{4 B^{2}+w^{2}}-y_{0}$, by (6) and from this value of $y_{03}$ we get the value of $y_{13}=y_{0}$.

Then, the two values

$$
y_{02}=y_{13}=y_{0} \quad \text { and } \quad y_{03}=y_{12}=\frac{8 A \delta}{4 B^{2}+w^{2}}-y_{0} \quad\left(\text { if there exists } y_{0}\right)
$$

are contribution to a same solution $\left(y_{02}, y_{12}\right)$ with $y_{02} \neq y_{12}$ for system (5). Consequently, they contribute to a same limit cycle. So the discontinuous piecewise linear differential system (44) has at most one limit cycle. Moreover, we can choose the appropriate parameters $a, b, c, d, B, A, \delta, \mu$ and $w$ in such a way that $f(y)=0$ has exactly two real roots $y_{02}$ and $y_{12}=\frac{8 A \delta}{4 B^{2}+w^{2}}-y_{0}$ that can provide at 1 limit cycle for the discontinuous piecewise linear differential system (4). 
Using the first integrals of both linear differential systems of (44) and knowing that the non-algebraic crossing periodic orbits pass through the points

$\left(0, y_{0}\right)$ and $\left(0, \frac{8 A \delta}{4 B^{2}+w^{2}}-y_{0}\right), \quad$ where $y_{0}$ is the zero of $f\left(y_{0}\right)=0$.

Thus these expressions are:

$$
\begin{aligned}
\Gamma= & \left\{\begin{array}{c}
(x, y) \in \Sigma_{+}: 4(A x+B y)^{2}+8 A(D x-\delta y)+w^{2} y^{2} \\
=\left(4 B^{2}+w^{2}\right) y_{0}^{2}-8 A \delta y_{0}
\end{array}\right\} \\
& \cup\left\{\begin{array}{c}
(x, y) \in \Sigma_{-}:((a+b \mu)(a x+b y)+a c+b d) e^{\frac{a+b \mu}{d-c \mu}(\mu x-y)} \\
=\left(b(a+b \mu) y_{0}+a c+b d\right) e^{\frac{a+b \mu}{c \mu d} y_{0}}
\end{array}\right\} .
\end{aligned}
$$

This completes the proof of Theorem 1.3 in Case 2.

\section{Proof of proposition 1.4}

We shall prove that the discontinuous piecewise linear differential system (3) has exactly one hyperbolic and non algebraic limit cycle. It is easy to see that the left half system has no equilibria, neither real nor virtual and since $\pm \frac{1}{2} i$ are the eigenvalues of the matrices of the right half system of (3), then this system has their equilibria as center type at the point $\left(-12, \frac{11}{4}\right)$.

The two linear differential systems of (3) have the following first integrals:

$$
\begin{aligned}
& H_{1}(x, y)=4\left(\frac{5}{16} x+y\right)^{2}+\frac{5}{2}(x+y)+y^{2}, \\
& H_{2}(x, y)=((1-\mu) x+y+1) e^{2 x \mu-2 y},
\end{aligned}
$$

in $\Sigma_{+}$and $\Sigma_{-}$, respectively.

Now we shall use the notation and the expressions of the proof of Theorem 1.3 . System (5) can be written as:

$$
\begin{array}{r}
y_{0}\left(2 y_{0}+1\right)-y_{1}\left(2 y_{1}+1\right)=0, \\
\left(y_{0}+1\right) e^{-2 y_{0}}-\left(y_{1}+1\right) e^{-2 y_{1}}=0 .
\end{array}
$$

Taking into account that we are only interested in the solutions $\left(y_{0}, y_{1}\right)$ satisfying $y_{1} \neq y_{0}$, the unique solution of the first equation of the previous system is

$$
y_{1}=-y_{0}-\frac{1}{2} \text {. }
$$




\section{NON ALGEBRAIC LIMIT CYCLE FOR A CLASS OF PIECEWISE LINEAR SYSTEMS}

Then, for the discontinuous piecewise linear differential system (3), the function (10) becomes

$$
f(y)=(y+1)+\left(y-\frac{1}{2}\right) e^{4 y+1} .
$$

The graph of this function is given in Figure 1.

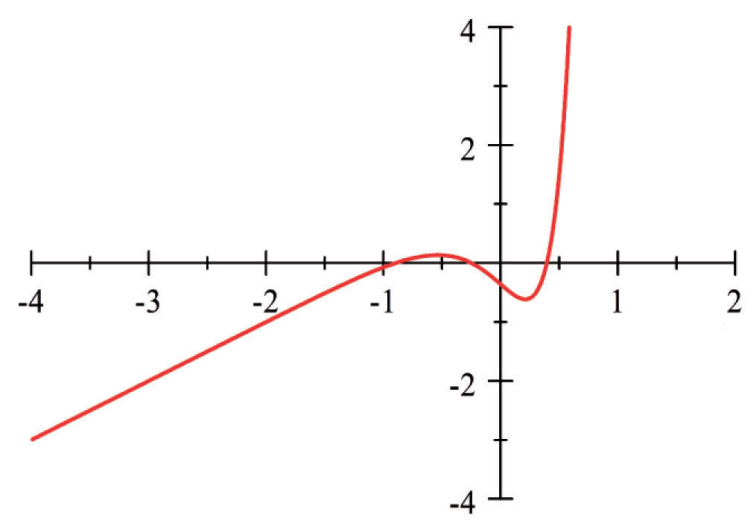

Figure 1. The graph of the function $f(y)$.

The three solutions of $f(y)=0$ are:

$$
y_{01}=-0.25, \quad y_{02}=-0.89392 \quad \text { and } \quad y_{03}=0.39392
$$

in the interval $\mathbb{R}$. From these values of $y_{0 i}$ we get values of

$$
y_{11}=y_{01}=-0.25, \quad y_{12}=0.39392 \quad \text { and } \quad y_{13}=-0.89392 \text {. }
$$

Consequently, the discontinuous piecewise linear differential system (3) has exactly one limit cycle.

Straightforward computations show that the solution passing through the crossing points $\left(0, y_{02}\right)$ and $\left(0, y_{12}\right)$ corresponds to:

$$
\begin{aligned}
\Gamma= & \left\{(x, y) \in \Sigma_{+}: 4\left(\frac{5}{16} x+y\right)^{2}+\frac{5}{2}(x+y)+y^{2}=1.7607\right\} \\
& \cup\left\{(x, y) \in \Sigma_{-}:(x+y-\mu x+1) e^{2 \mu x-2 y}=0.63399\right\} .
\end{aligned}
$$

Moreover, $\Gamma$ is non algebraic and is travelled in counter-clockwise sense around the sliding segment

$$
\Sigma_{r s}=\left\{(0, y) \in \Sigma:-\frac{1}{2} \leq y \leq \frac{-1}{4}\right\} .
$$




\section{AZIZA BERBACHE}

Now we shall prove that this non-algebraic crossing periodic orbit is a hyperbolic limit cycle. Let $T$ be the period of the periodic solution

$$
\Gamma:\{(x(t), y(t)), t \in[0, T]\} .
$$

To see that $\Gamma$ is in fact a hyperbolic limit cycle, we recall a classic result characterizing limit cycles among other periodic orbits for a smooth differential system in the plane (see for instance Perko [24] for more details), which means that $\Gamma(t)$ is a hyperbolic limit cycle when

$$
\int_{0}^{T} \operatorname{div}(\Gamma(t)) \mathrm{d} t \neq 0
$$

stable if $\int_{0}^{T} \operatorname{div}(\Gamma(t)) \mathrm{d} t<0$, and unstable if $\int_{0}^{T} \operatorname{div}(\Gamma(t)) \mathrm{d} t>0$. The formula (11) can be extended to the discontinuous piecewise linear differential systems. It is easy to check $\Gamma$ given by the analytic curves that $\left(x_{+}(t), y_{+}(t)\right)$ in the half-plane $\Sigma_{-}$for $t \in[0,1.6220]$, and by and $\left(x_{-}(t), y_{-}(t)\right)$ in the half-plane $\Sigma_{-}$ for $t \in[1.6220,2.5757]$, where

$$
\begin{aligned}
& x_{+}(t)=16 \cos \frac{1}{2} t-4 \cos \frac{1}{2} t-2 \sin \frac{1}{2} t-16-8 y_{02} \sin \frac{1}{2} t+4, \\
& y_{+}(t)=4+\frac{5}{4} \cos \frac{1}{2} t-4 \cos \frac{1}{2} t+2 D \sin \frac{1}{2} t+y_{02} \cos \frac{1}{2} t+2 y_{02} \sin \frac{1}{2} t-\frac{5}{4}, \\
& x_{-}(t)=\frac{1}{2} t-y_{12}+e^{-t}\left(y_{12}+1\right)-1, \\
& y_{-}(t)=y_{12}-\mu+t\left(\frac{1}{2} \mu-\frac{1}{2}\right)+e^{-t}\left(\mu+\mu y_{12}\right)-\mu y_{12} .
\end{aligned}
$$

Therefore, the periodic orbit $\Gamma$ has period $T=2.5757$. Then for the discontinuous piecewise linear differential system (3), we have

$$
\Gamma:\left\{\left(x_{+}(t), y_{+}(t)\right), t \in[0,1.6220]\right\} \cup\left\{\left(x_{-}(t), y_{-}(t)\right), t \in[1.6220, T]\right\},
$$

thus

$$
\int_{0}^{T} \operatorname{div}(\Gamma(t)) \mathrm{d} t=-\int_{1.6220}^{2.5757} \mathrm{~d} t=-0.9537<0 .
$$

Consequently, $\Gamma$ is a stable hyperbolic non algebraic limit cycle of the discontinuous piecewise linear differential system (3).

Example. Considering the parameter $\mu=-3$, we obtain the discontinuous piecewise linear differential system

$$
\begin{array}{lll}
\dot{x}=-x-4 y-1, & \dot{y}=\frac{5}{16} x+y+1 & \text { in } \Sigma_{+}, \\
\dot{x}=2 x-y-\frac{1}{2}, & \dot{y}=6 x-3 y-2, & \text { in } \Sigma_{-} .
\end{array}
$$




\section{NON ALGEBRAIC LIMIT CYCLE FOR A CLASS OF PIECEWISE LINEAR SYSTEMS}

Then, this system has exactly one explicit hyperbolic non algebraic crossing limit cycle $\Gamma$ that intersects the switching line $\Sigma$ at the two points

$$
y_{0}=-0.89392 \text { and } y_{1}=0.39392 \text {, }
$$

this limit cycle is given by

$$
\begin{aligned}
& \Gamma=\left\{(x, y t) \in \Sigma_{+}: 4\left(\frac{5}{16} x+y\right)^{2}+\frac{5}{2}(x+y)+y^{2}=1.7607\right\} \cup \\
& \left\{(x, y) \in \Sigma_{-}:(x+y+3 x+1) e^{-6 x-2 y}=0.63399\right\} \text {. }
\end{aligned}
$$

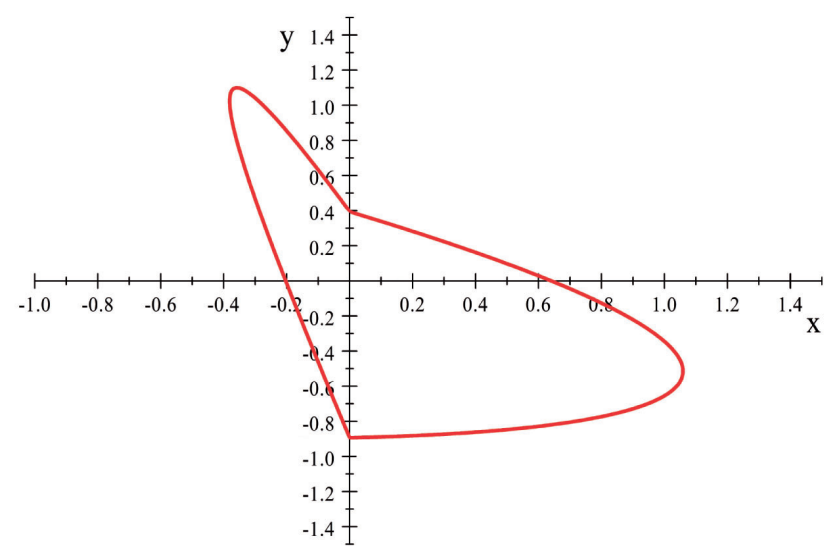

FIGURE 2. The limit cycle of the discontinuous piecewise linear differential system (12).

\section{REFERENCES}

[1] ARTÉS, J.C.-LLIBRE, J.-MEDRAdO, J.C.-TEIXEIRA, M. A.: Piecewise linear differential systems with two real saddles, Math. Comput. Simulation 95 (2014), 13-22.

[2] BERBACHE, A.: Two limit cycles for a class of discontinuous piecewise linear differential systems with two pieces, Sib. Élektron. Mat. Izv. 17 (2020), 1488-1515.

[3] BERBACHE, A.: Two explicit non-algebraic crossing limit cycles for a family of piecewise linear systems, Mem. Differ. Equ. Math. Phys. 83 (2021), 13-29.

[4] DE CARVALHO BRAGA, D. C.-MELLO, L. F.: Limit cycles in a family of discontinuous piecewise linear differential systems with two zones in the plane, Nonlinear Dynam. 73 (2013), 1283-1288.

[5] BUZZI, C.-PESSOA, C.-TORREGROSA, J.: Piecewise linear perturbations of a linear center, Discrete Contin. Dyn. Syst. 33 (2013), no. 9, 3915-3936.

[6] DI BERNARDO, M.-BUDD, C. J.-CHAMPNEYS, A.R.-KOWALCZYK, P.: Piecewise-Smooth Dynamical Systems. Theory and Applications. Appl. Math. Sci. Vol. 163, Springer-Verlag, New York, NY, 2008.

[7] EUZÉBIO, R.D.-LLIBRE, J.: On the number of limit cycles in discontinuous piecewise linear differential systems with two zones separated by a straight line, J. Math. Anal. Appl. 424 (2015), no. 1, 475-486. 


\title{
AZIZA BERBACHE
}

[8] FILIPPOV, A.F.: Differential equations with discontinuous right-hand sides, Mat. Sb. (N.S.), 51 (93) (1960), no. 1, 99-128.

[9] FREIRE, E.- PONCE, E.-RODRIGO, F.-TORRES, F.: Bifurcation sets of continuous piecewise linear systems with two zones, Int. J. Bifurcation and Chaos 8 (1998), 20732097.

[10] FREIRE, E.-PONCE E.-TORRES, F.: Canonical Discontinuous Planar Piecewise Linear Systems, SIAM J. Appl. Dynam. Syst. 11 (2012), 181-211.

[11] FREIRE, E.-PONCE E.-TORRES, F.: A general mechanism to generate three limit cycles in planar Filippov systems with two zones, Nonlinear Dynam. 78 (2014), no. 1, 251-263.

[12] HAN, M.-ZHANG, W.: On Hopf bifurcation in non-smooth planar systems, J. Differ. Equ. 248 (2010), 2399-2416.

[13] HUAN, S. M.-YANG,X.S.: On the number of limit cycles in general planar piecewise linear systems, Discrete Contin. Dynam. Syst. 32 (2012), 2147-2164.

[14] HUAN, S. M.-YANG, X.S.: On the number of limit cycles in general planar piecewise linear systems of node-node types, J. Math. Anal. Appl. 411 (2013), 340-353.

[15] HUAN, S. M.-YANG, X. S.: Existence of limit cycles in general planar piecewise linear systems of saddle-saddle dynamics, Nonlinear Anal. 92 (2013), 82-95.

[16] LI, L.: Three crossing limit cycles in planar piecewise linear systems with saddle-focus type, Electron. J. Qual. Theory Differ. Equ. 2014 (2014), paper no. 70, 14 p.

[17] LLIBRE, J.-PONCE, E.: Three nested limit cycles in discontinuous piecewise linear differential systems with two zones, Dynam. Contin. Discrete Impuls. Syst. Ser. B Appl. Algorithms 19 (2012), no. 3, 325-335.

[18] LLIBRE, J.-NOVAES, D. D.-TEIXEIRA, M. A.: Maximum number of limit cycles for certain piecewise linear dynamical systems, Nonlinear Dyn. 82 (2015), no. 3, 1159-1175.

[19] LLIBRE, M.-ORDÓÑEZ, M.- PONCE, E.: On the existence and uniqueness of limit cycles in planar piecewise linear systems without symmetry, Nonlinear Anal. Series B: Real World Appl. 14 (2013), no. 5, 2002-2012.

[20] LLIBRE, J.-TEIXEIRA, M. A.: Piecewise linear differential systems without equilibria produce limit cycles?, Nonlinear Dyn. 88 (2017), 157-164.

[21] LLIBRE, J.-ZHANG, X.: Limit cycles for discontinuous planar piecewise linear differential systems separated by one straight line and having a center, J. Math. Anal. Appl. 467 (2018), 537-549.

[22] LUM, R.-CHUA, L. O.: Global properties of continuous piecewise-linear vector fields. Part I: Simplest case in $\mathbb{R}^{2}$, Internat. J. Circuit Theory Appl. 19 (1991), 251-307.

[23] LUM, R.-CHUA, L. O.: Global properties of continuous piecewise-linear vector fields. Part II: Simplest symmetric case in $\mathbb{R}^{2}$, Internat. J. Circuit Theory Appl. 20 (1992), 9-46.

[24] PERKO, L.: Differential Equations and Dynamical Systems (3rd edition). In: Texts in Appl. Math. Vol. 7. Springer-Verlag, New York, 2001.

Received September 1, 2020

\author{
Department of Mathematics \\ Faculty of Mathematics and Informatics \\ University of Bordj Bou Arréridj \\ 34265 El Anasser \\ ALGERIA \\ E-mail: azizaberbache@hotmail.fr
}

